\title{
Deciphering the pharmacological mechanism of the Chinese formula Huanglian-Jie-Du decoction in the treatment of ischemic stroke using a systems biology-based strategy
}

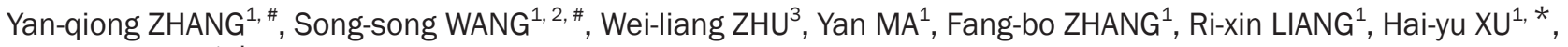 \\ Hong-jun YANG ${ }^{1, *}$ \\ ${ }^{1}$ Institute of Chinese Materia Medica, China Academy of Chinese Medical Sciences, Beijing 100700, China; ${ }^{2}$ School of Traditional \\ Chinese Medicine, Capital Medical University, Beijing 100069, China; ${ }^{3}$ Shanghai Institute of Materia Medica, Chinese Academy of \\ Sciences, Shanghai 201203, China
}

\begin{abstract}
Aim: Huanglian-Jie-Du decoction (HLJDD) is an important multiherb remedy in TCM, which is recently demonstrated to be effective to treat ischemic stroke. Here, we aimed to investigate the pharmacological mechanisms of HLJDD in the treatment of ischemic stroke using systems biology approaches.

Methods: Putative targets of HLDD were predicted using MetaDrug. An interaction network of putative HLJDD targets and known therapeutic targets for the treatment of ischemic stroke was then constructed, and candidate HLDD targets were identified by calculating topological features, including 'Degree', 'Node-betweenness', 'Closeness', and 'K-coreness'. The binding efficiencies of the candidate HLDD targets with the corresponding compositive compounds were further validated by a molecular docking simulation.

Results: A total of 809 putative targets were obtained for 168 compositive compounds in HLDD. Additionally, 39 putative targets were common to all four herbs of HLDD. Next, 49 major nodes were identified as candidate HLDD targets due to their network topological importance. The enrichment analysis based on the Gene Ontology (GO) annotation system and the Kyoto Encyclopedia of Genes and Genomes (KEGG) pathway demonstrated that candidate HLJDD targets were more frequently involved in G-protein-coupled receptor signaling pathways, neuroactive ligand-receptor interactions and gap junctions, which all played important roles in the progression of ischemic stroke. Finally, the molecular docking simulation showed that 170 pairs of chemical components and candidate HLDD targets had strong binding efficiencies.

Conclusion: This study has developed for the first time a comprehensive systems approach integrating drug target prediction, network analysis and molecular docking simulation to reveal the relationships between the herbs contained in HLDD and their putative targets and ischemic stroke-related pathways.
\end{abstract}

Keywords: Traditional Chinese Medicine; Chinese herbal formula; ischemic stroke; Huanglian-Jie-Du decoction; systems biology; network pharmacology

Acta Pharmacologica Sinica (2015) 36: 724-733; doi: 10.1038/aps.2014.124; published online 4 May 2015

\section{Introduction}

Ischemic stroke, one of the most common diseases that cause death and disability, is a major and increasingly important public health problem in both developed and developing countries worldwide ${ }^{[1]}$. This disease starts with the sudden

\footnotetext{
\#These authors contributed equally to this work.

* To whom correspondence should be addressed.

E-mail Hongjun0420@sina.com (Hong-jun YANG); hy_xu627@163.com (Hai-yu XU)

Received 2014-07-27 Accepted 2014-10-30
}

cessation of blood flow, oxygen, glucose, and energy in the lesion area, followed by a series of pathologic biochemical events called 'ischemic cascades', including glutamate-induced excitotoxicity, calcium influx, inflammation response, bloodbrain barrier breakdown, edema, and cell death ${ }^{[2]}$. Current therapeutic strategies for this disease are aimed at dissolving clots and restoring blood flow. Additional therapies that block excitatory neurotransmission, prevent the ischemic inflammatory response or scavenge free radicals have all shown promising therapeutic potential in animal stroke models ${ }^{[3]}$. With the advancement of preventive measures for controlling hyperten- 
sion, hypercholesterolemia, substance abuse, and smoking, as well as anticoagulation drugs, the incidence of ischemic stroke has been reduced ${ }^{[4]}$. However, there are no approved treatments that can effectively reduce stroke size or neurological disability in humans.

Traditional Chinese medicine (TCM) has been practiced for more than 2000 years in China. Its application to stroke therapy has a long history. The famous Chinese physician Zhongjing ZHANG described the symptoms of acute stroke approximately 2000 years ago. In 1995, the State Administration of TCM of the People's Republic of China issued standards for the diagnosis of stroke and the evaluation of the efficacy of treatments ${ }^{[5,6]}$. Herbal medicine therapy has been an alternative and promising strategy for the treatment of ischemic stroke. Huanglian-Jie-Du Decoction (HLJDD), also known as Oren-gedoku-to in Japanese, is an important multi-herb remedy in TCM. HLJDD, which is made according to the Handbook of Prescriptions for Emergency, is an aqueous extract of Rhizoma coptidis (Coptis chinensis Franch, Ranunculaceae), Radix scutellariae (Scutellaria baicalensis Georgi, Labiatae), Cortex phellodendri (Phellodendron amurense Rupr, Rutaceae), and, Fructus gardeniae (Gardenia jasminoides Ellis, Rubiaceae) at a ratio of 3:2:2:3. All the herbs in HLJDD are officially listed in the Chinese pharmacopoeia (Pharmacopoeia of PR China, 2010). Under the guidance of TCM theory, Rhizoma coptidis is the principal medicinal that can purge the heart and middle burner of fire; Radix scutellariae is the ministerial medicinal that can cleanse heat from the lung and eliminate fire from the upper burner; Cortex phellodendri is used to purge the lower burner of fire; and Fructus gardeniae purges fire from triple burners ${ }^{[7]}$. Recently, an increasing amount of evidence has revealed the pharmacological effects of this formula on inflammation, gastrointestinal disorders, diabetes, vasodilation, acute liver injury, Alzheimer disease, as well as in other cardiovascular diseases ${ }^{[8-10]}$. More specifically, research on the pharmacological and biochemical actions of HLJDD extract and its constituents has also demonstrated that it has protective effects against ischemic stroke. For example, Yun et al ${ }^{[11]}$ indicated that HLJDD could be effective in subjects with poststroke pathological laughter diagnosed as Yang Excess patterns and pattern identification; Hwang et al ${ }^{[12]}$ showed that HLJDD and its constituents could reduce ischemia-reperfusion brain injury and neutrophil infiltration in rats; and Kiga et al ${ }^{[13]}$ also indicated that HLJDD could suppress the onset of stroke in stroke-prone spontaneously hypertensive rats. However, the pharmacological mechanisms of the actions of HLJDD on ischemic stroke have not been fully elucidated due to a lack of appropriate methods.

With the development of systems biology, network biology, and polypharmacology, network pharmacology has been advocated by Andrew L Hopkins ${ }^{[14]}$. This novel research field is involved in the application of omics- and systems biologybased technologies ${ }^{[15]}$. It determines the synergistic effects and the underlying mechanisms of multi-component and multi-target agents by analyzing various networks of complex and multi-level interactions ${ }^{[16,17]}$. There are two types of approaches in network pharmacology: (1) bottom-up: the addition of well-known molecular drugs and the observation of synergistic effects; and (2) top-down: the reduction of a more general formula to the minimal elements that maintain its beneficial properties ${ }^{[18,19]}$. As a major tool in network pharmacology, network analysis based on widely existing databases allow us to form an initial understanding of mechanisms of action within the context of systems-level interactions. Because TCM herbal formulas are considered multi-component and multi-target therapeutics that potentially meet the demands of treating a number of complex diseases in an integrated manner, the methodologies of network pharmacology are suitable for pursuing a priori knowledge about the combinations of rules embedded in these formulas ${ }^{[20]}$. Thus, in this study, we developed a comprehensive systems approach for understanding the pharmacological mechanisms of the effects of HLJDD on ischemic stroke. Our protocol included three main steps: (1) the prediction of putative targets of HLJDD; (2) an investigation into the relationships between putative targets of HLJDD and ischemic stroke-imbalanced networks and related signaling pathways, which offers a great opportunity for a deep understanding of the pharmacological mechanisms of HLJDD on reversing this disease-related imbalanced network; (3) a validation of the binding efficiency of candidate HLJDD targets to the corresponding compositive compounds contained in this formula by molecular docking simulation. Figure 1 depicts a flowchart of the entire experimental procedure.

\section{Materials and methods}

\section{Data preparation}

\section{Compositive compounds of each herb contained in HLJDD}

Compositive compounds of each herb contained in HLJDD were obtained from a chemistry database ${ }^{[16]}$ (http://www. organchem.csdb.cn/scdb/main/slogin.asp, updated on 201405-05) that is specifically designed to store chemical information, including chemical and crystal structures, spectra, reactions, syntheses, and thermophysical data. We collected structural information for 21 Rhizoma coptidis compounds, 47 Radix scutellariae compounds, 54 Cortex phellodendri compounds, and 78 Fructus gardeniae compounds. Detailed information about these compositive compounds of each herb contained in HLJDD is described in Supplementary Table S1. The molecular files of all the compositive compounds were downloaded from ChemSpider (http:/ / www.chemspider. com/, updated on 2011-12-23) and were saved to a .sdf format.

\section{Known therapeutic targets for the treatment of ischemic stroke}

Known therapeutic targets for the treatment of ischemic stroke were obtained from two resources. The first was the DrugBank database ${ }^{[21]}$ (http:/ / www.drugbank.ca/, version: 3.0). We only used drug-target interactions involving drugs that are FDA-approved therapeutic targets for the treatment of ischemic stroke and whose targets are human genes/proteins. In total, we obtained 70 known therapeutic targets for the treatment of ischemic stroke. The second resource was 


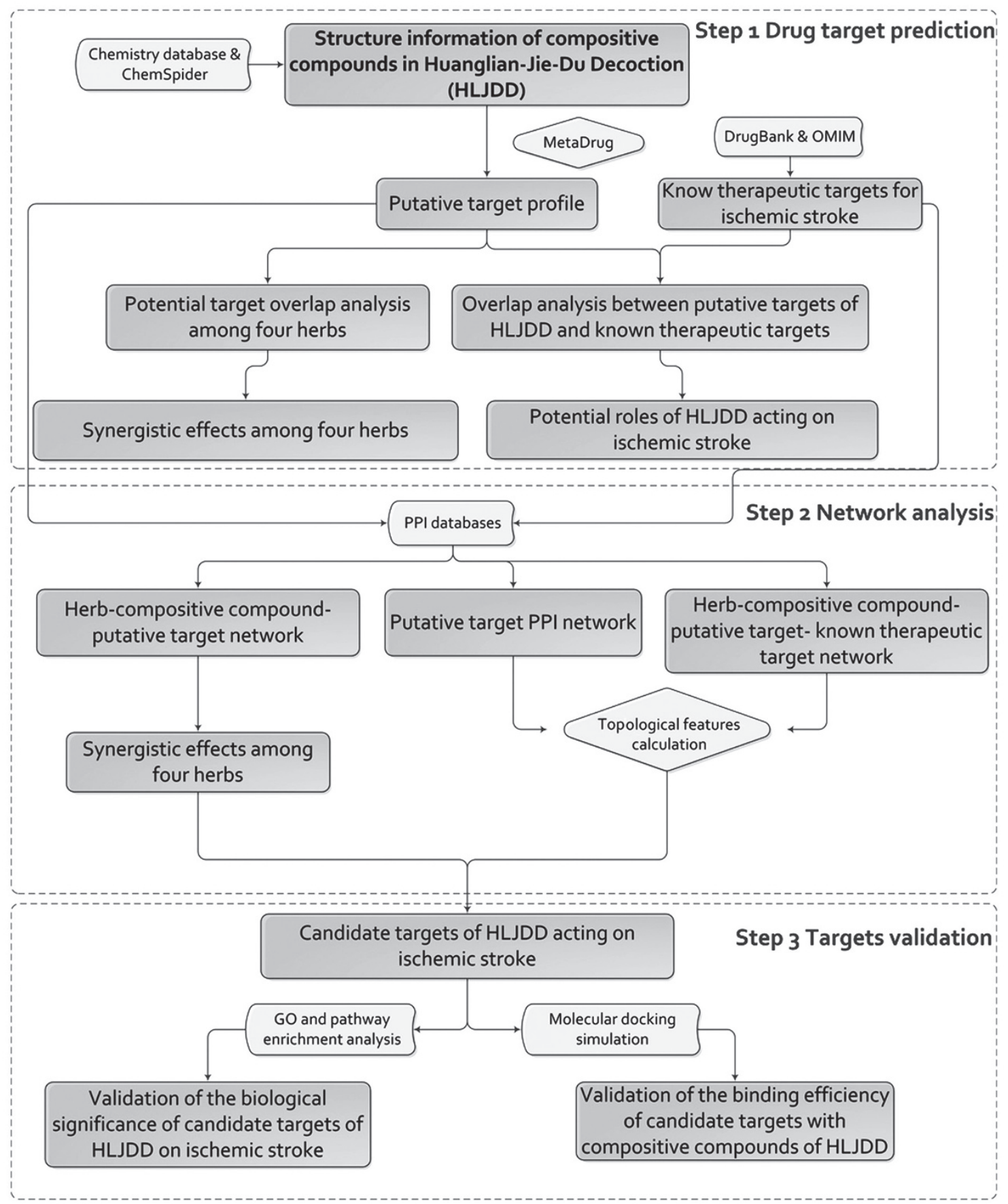

Figure 1. A schematic diagram of the systems biology-based strategies for determining the pharmacological mechanisms of action of the herbal formula HLDDD on ischemic stroke.

the Online Mendelian Inheritance in Man (OMIM) database ${ }^{[22]}$ (http://www.omim.org/, last updated: October 31, 2013). We searched the OMIM database with the keyword 'ischemic stroke' and found 72 known therapeutic targets for the treatment of ischemic stroke. Detailed information about these known therapeutic targets is described in Supplementary Table S2. After removing redundancy, there were 62 known therapeutic targets for the treatment of ischemic stroke col- lected in this study.

\section{Protein-protein interaction (PPI) data}

PPI data were imported from eight existing PPI databases, including the Human Annotated and Predicted Protein Interaction Database (HAPPI) ${ }^{[23]}$, Reactome ${ }^{[24]}$, Online Predicted Human Interaction Database (OPHID) ${ }^{[25]}$, InAct ${ }^{[26]}$, Human Protein Reference Database (HPRD) ${ }^{[27]}$, Molecular Interaction 
Database $(\mathrm{MINT})^{[28]}$, Database of Interacting Proteins (DIP) ${ }^{[29]}$, and PDZBase ${ }^{[30]}$. Detailed information about these PPI databases is described in Supplementary Table S3.

\section{Drug target prediction for HLDD}

The putative targets of the HLJDD compositive compounds were predicted by MetaDrug from GeneGo, Inc ${ }^{[31]}$. MetaDrug combines chemical structural analysis tools, a structureactivity database, a systems biology database of molecular interactions, canonical signaling and metabolic pathways and gene-biological property associations. MetaDrug uses three methods to predict putative targets of certain compounds. The first method uses the MetaBase database, which contains compound-protein interactions. This database allows compounds with known biological activities to be directly incorporated into networks and their pharmacological properties to be further investigated. The second method uses QSAR predictions of protein target affinity from the included models that define a limited number of potential targets for novel molecules and/or their metabolites submitted for analysis. The third method performs a similarity search for the structure and its major metabolites against a database of existing structures and their targets. Putative targets of certain compounds are inferred through structurally similar compounds in the database (GeneGo, personal communication). MetaDrug was developed with an Oracle version 9.2.0.4 Standard Editionbased architecture (Oracle, Redwood Shores, CA, USA). The software runs on an Intel-based 32-bit server running RedHat Linux Enterprise 3 AS (RedHat, Raleigh, NC, USA), and the web server runs Apache 1.3.x/mod_perl (http://perl.apache. org/start/ index.html).

\section{Network construction and analysis}

The herbs, compositive compounds, putative targets of HLJDD and known therapeutic targets for the treatment of ischemic stroke were used to construct an herb-compositive compoundputative target network, a putative target PPI network, and an herb-compositive compound-putative target-known therapeutic target network, respectively. The PPI data were obtained from eight existing PPI databases, as mentioned above. Then, we applied Navigator software (Version 2.2.1, Toronto, ON, USA) and Cytoscape (Version 2.8.1, Boston, MA, USA) to visualize the networks.

\section{HLJDD herb-compositive compound-putative target-known} ischemic stroke target network

The HLJDD herb-compositive compound-putative targetknown ischemic stroke target network was constructed by linking four herbs contained in HLJDD, the compositive compounds contained in all the herbs, their putative targets, and the known ischemic stroke targets that interact with the putative targets. According to a previous study by $\mathrm{Li}$ et al $^{[32]}$, we identified a node as a hub protein if its degree was more than 2 -fold the median degree of all the nodes in a network. Then, the PPIs among the hub protein targets were used to construct the hub putative target PPI network. Moreover, four topologi- cal features, 'Degree', 'Node betweenness', 'Closeness', and ' $K$ value' (defined in the 'Defining features set' section), were chosen to identify the major putative targets, which had values of the four features that were higher than the corresponding median values.

\section{Defining features set}

For each node $i$ in the above networks, we defined four measures for assessing its topological property. (1) 'Degree' was defined as the number of links to node i. (2) 'Node betweenness' was defined as the number of the shortest paths between pairs of nodes that ran through node i. (3) 'Closeness' was defined as the inverse of the farness, which was the sum of the node i distances to all other nodes. The closeness centrality can be regarded as a measure of how long it will take to sequentially spread information from node $i$ to all the other nodes. Degree, node betweenness and closeness centralities can be used to measure a protein's topological importance in the network. The larger a protein's degree/node betweenness/closeness centrality, the more important that protein is in the PPI network ${ }^{[33]}$. (4) K-core analysis is an iterative process in which the nodes are removed from the networks in the order of the least connected ${ }^{[34]}$. The core of maximum order is defined as the main core or the highest k-core of the network. A k-core sub-network of the original network can be generated by recursively deleting vertices from the network whose degree is less than $\mathrm{k}$. This results in a series of sub-networks that gradually reveal the globally central region of the original network. On this basis, ' $K$ value' is used to measure the centrality of node $\mathrm{i}$.

Gene Ontology (GO) and pathway enrichment analysis for major putative targets of HLJDD for the treatment of ischemic stroke

We used the Database for Annotation, Visualization and Integrated Discovery ${ }^{[35]}$ (DAVID, http://david.abcc.ncifcrf.gov/ home.jsp, version 6.7) for the GO enrichment analysis. We also performed a pathway enrichment analysis using pathway data obtained from the FTP service of Kyoto Encyclopedia of Genes and Genomes ${ }^{[36]}$ (KEGG, http:/ / www.genome.jp/ kegg/, last updated: Oct 16, 2012).

\section{Molecular docking simulation}

A molecular docking simulation was performed to validate the binding efficiency of candidate targets to compositive compounds of each herb contained in HLJDD using the program LibDock implemented in Discovery Studio 2.5 (DS 2.5). All the crystal structures of the targets were directly downloaded from the RCSB protein data bank (http://www.pdb.org/, updated on 2014-3-11) and were carefully checked for their resolutions. A docking score calculated by the customizable scoring function of LibDock was used to measure the binding efficiency of each candidate target to the corresponding compound. The docking scores of the candidate targets that could bind their corresponding compounds with a strong binding efficiency were higher than 100 , which was the median value of all the docking scores. 


\section{Results}

Chemical information about HLJDD and the prediction of its potential targets

HLJDD, as a classic Chinese herbal prescription, has attracted wide attention from researchers. Many phytochemical constituents were chemically separated, structurally identified from four single herbs, and systematically collected into the chemistry database of TCM. Recently, many analytical techniques, including capillary electrophoresis $(\mathrm{CE})^{[37]}$, HPLC-DAD ${ }^{[38]}$, and HPLC-Q-Exactive ${ }^{[39]}$, have been applied for qualitative and quantitative analysis of the chemical profiles of HLJDD. In particular, the feasible and accurate method of HPLC combined with hybrid quadrupole-orbitrap and triple-quadrupole mass spectrometry has been used to rapidly clarify and quantify the chemical profile of HLJDD. As a result, 69 compounds, including iridoids, alkaloids, flavonoids, triterpenoid, monoterpene, and phenolic acids, have been identified by their characteristically high-resolution mass data, which provided the basic chemical information for our study.

Following the drug target prediction by Metadrug ${ }^{[31]}, 809$ putative targets were obtained for 168 compositive compounds contained in HLJDD after deleting redundancy. On average, each compound had 4.82 putative targets. Detailed information about the predicted drug targets of HLJDD is described in Supplementary Table S4.

In addition, 39 putative targets were common to all four herbs contained in HLJDD. We found that there were different numbers of common putative targets between any two herbs (Table 1), suggesting that these herbs might play a role in some of the same biological processes or pathways. More interestingly, the adjunctive herbs Cortex phellodendri and Fructus gardeniae both shared more common putative targets with the primary herb Rhizoma coptidis (114/149, 76.51\% and $74 / 149,49.66 \%$, respectively; Table 1 ) than with the ministerial herb Radix scutellariae $(409 / 488,83.81 \%$ and $448 / 488,91.80 \%$, respectively; Table 1), suggesting their roles in facilitating the effects of other herbs.

Identification of the underlying pharmacological mechanisms of the actions of HLJDD on ischemic stroke

To elucidate the pharmacological mechanisms of the actions of HLJDD on ischemic stroke, we constructed an herb-compositive compound-putative target network, a putative target PPI network, and an herb-compositive compound-putative targetknown therapeutic target network.
Herb-compositive compound-putative target network for HLDD

We first constructed the herb-compositive compound-putative target network for HLJDD to clarify the relationships between the herbs and the corresponding putative targets. As shown in Figure 2, the network consists of 1100 nodes (4 ingredients, 286 compositive compounds, and 810 putative targets) and 6996 edges. The mean number of putative targets per compositive compound was 2.83. Among 286 compositive compounds, 8 had high-degree distributions, and each of them hit 331 putative targets. Three of them come from Radix scutellariae, such as stigmasterol, quebrachol, and campesterol; three of them come from Cortex phellodendri, such as quebrachol, campesterol, and stigmasterol; and the other two (quebrachol and stigmasterol) come from Fructus gardeniae. These compositive compounds with the highest degree in the network may play important roles in this pharmacological network.

\section{HLJDD herb-compositive compound-putative target-known} ischemic stroke target network

Then, we constructed the HLJDD herb-compositive compound-putative target-known ischemic stroke target network to evaluate the importance of putative targets in reversing the imbalanced network of ischemic stroke. PPI data between putative targets and known therapeutic targets for the treatment of ischemic stroke were collected from existing PPI databases (as described in the Materials and methods section). As shown in Figure 3A, the network consists of 788 nodes (containing 4 ingredients and 286 compositive compounds contained in HLJDD, as well as 436 putative targets and 62 known therapeutic targets for the treatment of ischemic stroke) and 6491 edges. Four topological features, 'Degree', 'Node betweenness', 'Closeness', and 'K value' (defined in the Materials and methods section), were chosen to identify the major nodes in this network. In total, we identified 77 major nodes containing 26 compositive compounds contained in HLJDD, 49 putative targets, 2 known therapeutic targets for the treatment of ischemic stroke. The 'Degree', 'Node betweenness', 'Closeness', and ' $K$ value' of these nodes were all larger than the corresponding median values. For detailed information about the topological features of the 77 major nodes in this network, please see Supplementary Table S5.

To further clarify the pharmacological mechanisms of the actions of HLJDD on ischemic stroke, we constructed major compositive compound-major putative target-known therapeutic targets for the treatment of ischemic stroke network

Table 1. Potential target overlaps among five ingredients of HLJDD.

\begin{tabular}{lcccc}
\hline \multicolumn{1}{c}{ Ingredients } & Rhizoma coptidis (149) & Radix scutellariae (488) & Cortex phellodendri (607) & Fructus gardeniae (608) \\
\hline Rhizoma coptidis (149) & - & 50 & 114 & 74 \\
Radix scutellariae (488) & 50 & - & 409 & 448 \\
Cortex phellodendri (607) & 114 & 409 & - & 445 \\
Fructus gardeniae (608) & 74 & 448 & $445^{\mathrm{b}}$ \\
\hline
\end{tabular}


A

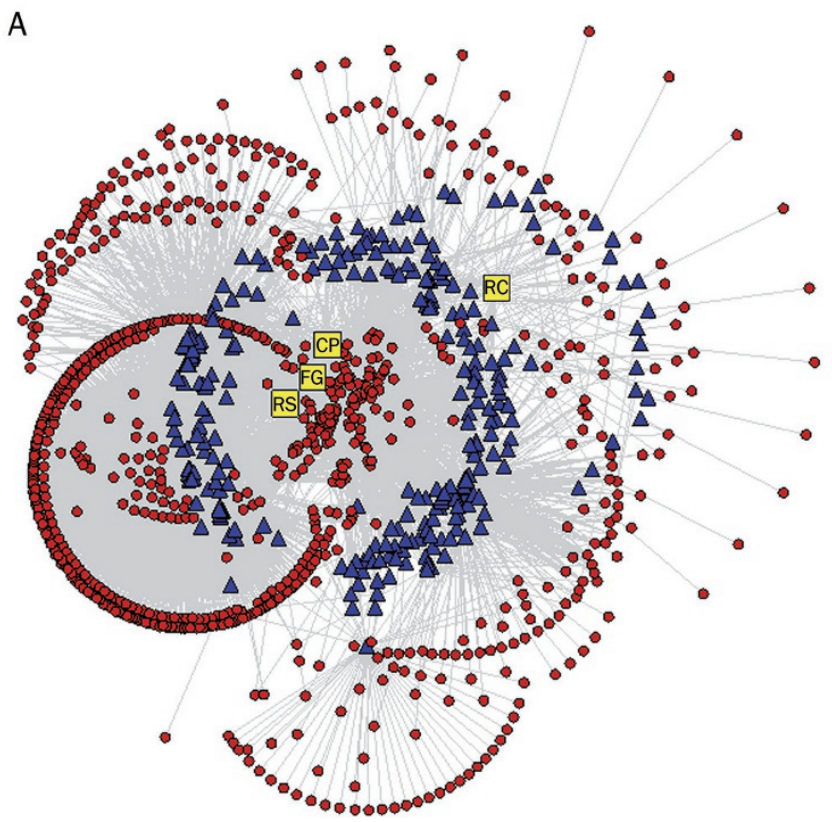

B

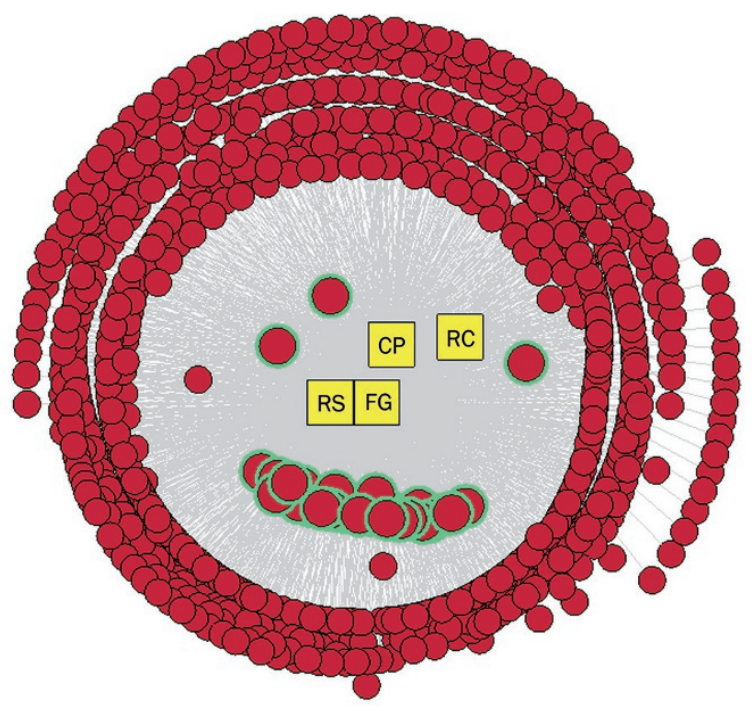

Figure 2. Interaction network between compositive compounds of HLDD and their putative targets, which was built and visualized with Navigator. (A) Multi-level network of herb-compositive compounds-putative target; (B) Interaction network of herb-putative target extracted from (A) to understand the relationships among the herbs in HLJDD. Edges: interactions between compositive compounds of HLDD and their putative targets; yellow square nodes: four herbs in HLJDD, including Rhizoma coptidis (RC), Radix scutellariae (RS), Cortex phellodendri (CP) and Fructus gardeniae (FG); blue triangular nodes: compositive compounds of HLJDD; red round nodes: putative targets of compositive compounds of HLJDD. Red round nodes with green rings: putative targets shared by four herbs in HLDD.

using the direct interactions among the 77 major nodes mentioned above. As shown in Figure 3B, this network contains 77 nodes (including 26 compositive compounds contained in HLJDD, 49 putative targets, and 2 known therapeutic targets for the treatment of ischemic stroke) and 693 edges. All the major compositive compounds in this network shared more than 6 major putative targets in this network, and $3,5,7,2^{\prime}, 6^{\prime}-5$-hydroxyflavones (Hq12) and quercetin (Zz44) had the largest number of common putative targets $(n=22$, Supplementary Table S6).

According to the results of an enrichment analysis based on the GO annotation system and the KEGG pathway, the major putative targets of the effects of HLJDD on ischemic stroke were frequently involved in various biological process and signaling pathways, such as G-protein-coupled receptor (GPCR) signaling pathways, neuroactive ligand-receptor interactions and gap junctions (Table 2). GPCRs perceive many extracellular signals and transduce them to heterotrimeric $G$ proteins, which further transduce these signals intracellularly to the appropriate downstream effectors ${ }^{[40]}$. GPCRs regulate vital processes by controlling the expression and function of seventransmembrane receptors, which are particularly critical for some widespread neurological disorders. For example, chemokine CXC ligand 12 (CXCL12) works in conjunction with the G-protein-coupled receptor CXCR4, which is found on the surface of a variety of cells, including neurons, astrocytes, microglia, bone marrow-derived cells, and other progenitor cells. CXCL12/CXCR4 may play important roles in multiple processes after ischemic stroke, which include inflammatory responses, focal angiogenesis, and the recruitment of bone marrow-derived cells and neural progenitor cells to the site of injury ${ }^{[4]]}$. In addition to their roles in stroke pathology, several GPCRs are thought to be key regulators of stroke repair. For example, CB1 and CB2, which belong to the GPCRs family, have been identified and are targeted by numerous exogenous and endogenous ligands. Under certain conditions, the activation of CB1 receptors in cerebral blood vessels can have beneficial anti-ischemic effects ${ }^{[42]}$. In the mammalian $\mathrm{CNS}$, direct intercellular communication between neighboring cells occurs through specialized structures known as gap junctions. A gap junction is a channel between two cells with a pore $\sim 2 \mathrm{~nm}$ in diameter, which allows the direct diffusion of ions and small molecules ${ }^{[43]}$. Recent studies have demonstrated that gap junctions may play important roles during neuronal development, ischemic stroke, trauma, and epilepsy ${ }^{[44]}$. However, the exact involvement of gap junctions in ischemia remains controversial. Several reports have indicated that astrocytic gap junctions could spread hypoxic injury, while other reports have demonstrated that blocking astrocytic gap junctions might increase neuronal death ${ }^{[45]}$. Kozoriz et al ${ }^{[46]}$ recently found that the gap junction protein connexin 43 may play a crucial role in neuroprotection in experimental stroke models. Reducing the expression of this protein in astrocytes may enhance injury upon middle cerebral artery occlusion. On this basis, the 


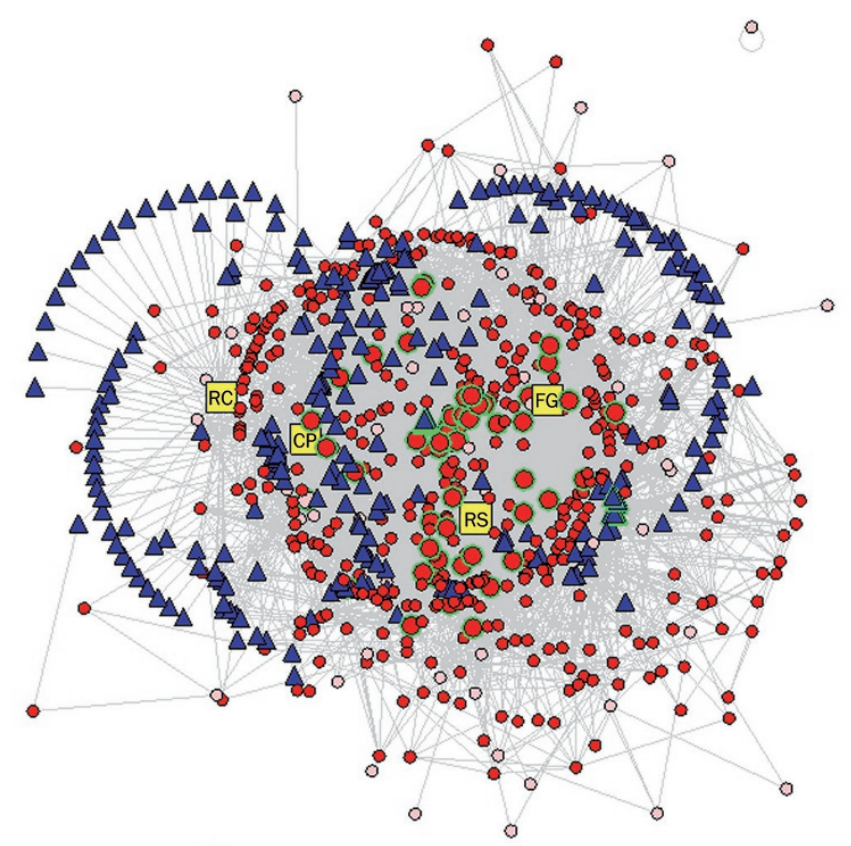

B

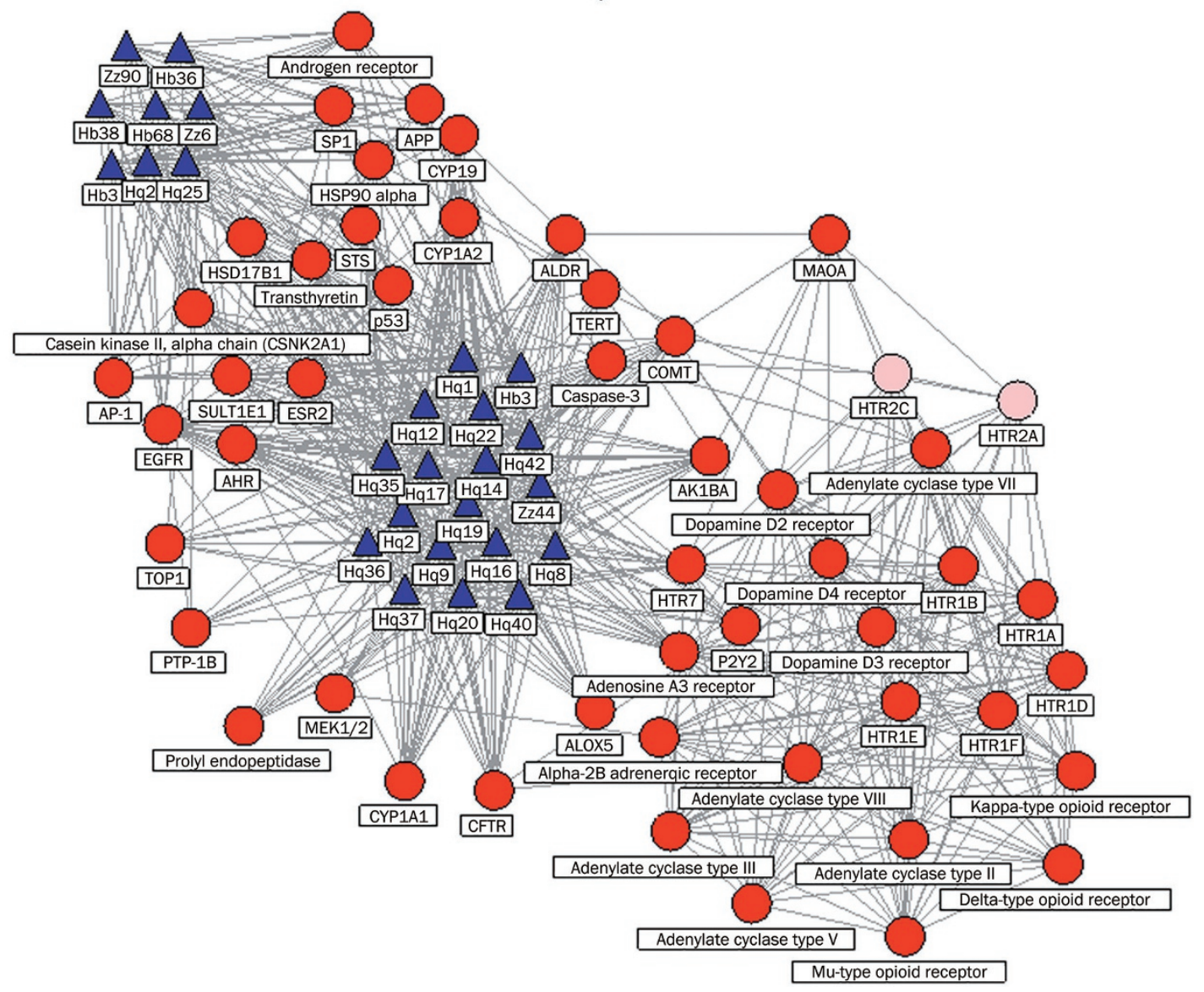

Figure 3. Network analysis for the identification of the underlying pharmacological mechanisms of the actions of HLDD acting on ischemic stroke (A) HLJDD herb-compositive compound-putative target-known ischemic stroke target network built and visualized with Navigator. (B) Major compositive compound-major putative target-known ischemic stroke target network built and visualized with Navigator. Edges: interactions among four herbs, compositive compounds, putative targets and known therapeutic targets for the treatment of ischemic stroke. Yellow square nodes: four herbs in HLJDD, including Rhizoma coptidis (RC), Radix scutellariae (RS), Cortex phellodendri (CP), and Fructus gardeniae (FG); blue triangular nodes: compositive compounds of HLDD; red round nodes: putative targets of compositive compounds of HLJDD; pink round nodes: known therapeutic targets for the treatment of ischemic stroke; nodes marked with green rings: major nodes, the 'Degree', 'Node betweenness', 'Closeness', and ' $K$ value' of which were all larger than the corresponding median values. 
Table 2. Top $10 \mathrm{GO}$ items of biological processes and top $10 \mathrm{KEGG}$ pathways significantly associated with the major putative targets of HLDD.

\begin{tabular}{|c|c|c|}
\hline Term & Counts & $P$-value \\
\hline \multicolumn{3}{|l|}{ Biological processes } \\
\hline GO:0007187 G-protein signaling, coupled to cyclic nucleotide second messenger & 17 & $1.02 \times 10^{-22}$ \\
\hline GO:0042221 response to chemical stimulus & 29 & $2.65 \times 10^{-22}$ \\
\hline GO:0019935 cyclic-nucleotide-mediated signaling & 17 & $8.02 \times 10^{-22}$ \\
\hline GO:0030817 regulation of cAMP biosynthetic process & 15 & $9.10 \times 10^{-20}$ \\
\hline G0:0030808 regulation of nucleotide biosynthetic process & 15 & $2.49 \times 10^{-19}$ \\
\hline GO:0030799 regulation of cyclic nucleotide metabolic process & 15 & $2.49 \times 10^{-19}$ \\
\hline GO:0006140 regulation of nucleotide metabolic process & 15 & $2.49 \times 10^{-19}$ \\
\hline G0:0007193 inhibition of adenylate cyclase activity by G-protein signaling & 12 & $1.59 \times 10^{-19}$ \\
\hline \multicolumn{3}{|l|}{ KEGG pathways } \\
\hline hsa04020:Calcium signaling pathway & 8 & $4.75 \times 10^{-5}$ \\
\hline hsa04914:Progesterone-mediated oocyte maturation & 7 & $3.74 \times 10^{-4}$ \\
\hline hsa04114:Oocyte meiosis & 7 & $1.88 \times 10^{-4}$ \\
\hline hsa04916:Melanogenesis & 6 & $4.22 \times 10^{-4}$ \\
\hline hsa04270:Vascular smooth muscle contraction & 6 & $9.69 \times 10^{-4}$ \\
\hline hsa00140:Steroid hormone biosynthesis & 5 & 0.001686 \\
\hline hsa05210:Colorectal cancer & 5 & 0.004028 \\
\hline
\end{tabular}

major putative targets of HLJDD that are significantly associated with these biological processes and pathways might play a role in the progression of ischemic stroke.

\section{Molecular docking validation}

The computational docking technique, as a structure-based method, is an invaluable tool in drug discovery and design. This technique can help researchers discover the relationship between the constituents of TCM and network targets ${ }^{[47]}$. DS LibDock is a powerful tool for high-throughput molecular docking $^{[48]}$. LibDock scoring is a fast, simple method to rapidly predict the binding affinity of a ligand, based on the geometry of a candidate ligand docked into a target receptor structure using empirical functions ${ }^{[49]}$. In this research, a docking score greater than 100 indicated a strong binding capacity between the components of HLJDD and the molecular targets. Detailed information about the docking scores of the candidate targets binding to their corresponding compounds contained in HLJDD is described in Supplementary Table S7. There were 169 pairs of compound-candidate effector molecule interactions with strong binding free energy. To investigate the regulatory effects of these compounds on the corresponding candidate effectors, we collected known drugs with similar structures to these compounds and their experimentally validated interactions with the corresponding candidate effectors using MetaDrug from GeneGo, Inc. Because drugs with similar structures often have similar functions, the regulatory effects of compounds on candidate effectors of HLJDD may be similar to those of the corresponding similar known drugs. As a result, there were 11 pairs of compound-candidate effector molecule interactions with positive effects and 114 pairs of compound-candidate effector molecule interactions with negative effects; these findings should be validated by further biological or biochemical experiments. Detailed information about the regulatory effects of similar known drugs on candidate targets of HLJDD is described in Supplementary Table S8.

\section{Discussion}

Accumulating evidence suggests that multi-target therapeutics are a promising paradigm for drug discovery and are expected to produce greater levels of efficacy with fewer adverse effects and toxicity than monotherapies. Chinese herbal formulas are characterized by multi-components and multi-targets. Systems biology-based approaches provide powerful tools for finding and elucidating active multi-components and their mechanisms of action. In the current study, we developed a comprehensive systems approach that integrated drug target prediction, network analysis, and target validation to reveal the relationships between the ingredients in HLJDD and their putative targets and ischemic stroke-related pathway systems for the first time. Our results show the following:

(1) The putative targets of four herbs in HLJDD were predicted and provided clues to investigate the pharmacological mechanisms of HLJDD for the treatment of ischemic stroke;

(2) The pharmacological network of HLJDD was built according to the relationships among herbs, chemical compo- 
nents, and putative targets, providing insights into the synergetic effects among the herbs contained in this formula.

(3) A multilevel network with a combination of an ischemic stroke-related imbalanced network and a pharmacological network of HLJDD was built and indicated the key players in this formula that act on ischemic stroke and the corresponding putative targets.

(4) According to the molecular docking simulation, 170 pairs of chemical components and putative targets had strong binding efficiencies.

However, this preliminary study, which was performed according to our strategy, was based on a bioinformatics analysis. Thus, further experimental studies are required to test these hypotheses.

Taken together, our data may support further assessments of the clinical application of HLJDD and may enable further research on TCM formulas in a more timely and cost-effective manner.

\section{Acknowledgements}

Financial support was provided by the Key Project of Chinese National Programs for Fundamental Research and Development (973 Program Grant № 2014CB543003), the Fundamental Research Funds for the Central Public Welfare Research Institutes (ZZ070831, ZZ070819), and the Beijing Joint Project Specific Funds and independent topics supported by Operational Expenses for Basic Research of China Academy of Chinese Medical Sciences (№ Z02063).

\section{Author contribution}

Yan-qiong ZHANG, Hai-yu XU, and Hong-jun YANG designed the research; Yan-qiong ZHANG and Song-song WANG performed the research and wrote the paper; Weiliang ZHU contributed analytic tools; Yan MA, Fang-bo ZHANG and Ri-xin LIANG analyzed the data.

\section{Supplementary information}

Supplementary information is available at Acta Pharmacologica Sinica's website.

\section{References}

1 Manning NW, Campbell BC, Oxley TJ, Chapot R. Acute ischemic stroke: time, penumbra, and reperfusion. Stroke 2014; 45: 640-4.

2 Ni X, Liu S, Guo X. Medium- and long-term efficacy of ligustrazine plus conventional medication on ischemic stroke: a systematic review and meta-analysis. J Tradit Chin Med 2013; 33: 715-20.

3 Singh P, Kaur R, Kaur A. Endovascular treatment of acute ischemic stroke. J Neurosci Rural Pract 2013; 4: 298-303.

4 Tymianski M. Novel approaches to neuroprotection trials in acute ischemic stroke. Stroke 2013; 44: 2942-50.

5 Fu DL, Lu L, Zhu W, Li JH, Li HQ, Liu AJ, et al. Xiaoxuming decoction for acute ischemic stroke: a systematic review and meta-analysis. J Ethnopharmacol 2013; 148: 1-13.

6 Chen YF. Traditional Chinese herbal medicine and cerebral ischemia. Front Biosci (Elite Ed) 2012; 4: 809-17.

7 Wu Y, Sun J, Shi R, Zhang A. Effect of Huanglian Jiedu tang active fraction on calcium overloading in neurons and related mechanism analysis. Zhongguo Zhong Yao Za Zhi 2010; 35: 2166-70. Chinese.

8 Zhang XJ, Deng YX, Shi QZ, He MY, Chen B, Qiu XM. Hypolipidemic effect of the Chinese polyherbal Huanglian Jiedu decoction in type 2 diabetic rats and its possible mechanism. Phytomedicine 2014; 21 : 615-23.

9 Ma YL, Li T, Wang BB, Jia B, Chen B, Su J, et al. Protection of huanglian jiedu decoction on livers of hyperlipidemia mice. Zhongguo Zhong Xi Yi Jie He Za Zhi 2013; 33: 1107-11. Chinese.

10 Feng YB, Luo WQ, Zhu SQ. Explore new clinical application of Huanglian and corresponding compound prescriptions from their traditional use. Zhongguo Zhong Yao Za Zhi 2008; 33: 1221-5. Chinese.

11 Yun SP, Jung WS, Park SU, Moon SK, Park JM, Ko CN, et al. Hwangryunhaedogtang (huanglianjiedutang) treatment for pathological laughter after stroke and importance of patterns identification: a preliminary study. Am J Chin Med 2007; 35: 725-33.

12 Hwang YS, Shin CY, Huh Y, Ryu JH. Hwangryun-Hae-Dok-tang (Huanglian-Jie-Du-Tang) extract and its constituents reduce ischemiareperfusion brain injury and neutrophil infiltration in rats. Life Sci 2002; 71: 2105-17.

13 Kiga C, Nakagawa T, Koizumi K, Sakurai H, Shibagaki Y, Ogawa K, et al. Expression patterns of plasma proteins in spontaneously diabetic rats after oral administration of a Kampo medicine, Hachimi-jio-gan, using SELDI ProteinChip platform. Biol Pharm Bull 2005; 28: 1031-7.

14 Hopkins AL. Network pharmacology: the next paradigm in drug discovery. Nat Chem Biol 2008; 4: 682-90.

15 Liang X, Li H, Li S. A novel network pharmacology approach to analyse traditional herbal formulae: the Liu-Wei-Di-Huang pill as a case study. Mol Biosyst 2014; 10: 1014-22.

16 Tao W, Xu X, Wang X, Li B, Wang Y, Li Y, et al. Network pharmacologybased prediction of the active ingredients and potential targets of Chinese herbal Radix Curcumae formula for application to cardiovascular disease. J Ethnopharmacol 2013; 145: 1-10.

17 Liu ZH, Sun XB. Network pharmacology: new opportunity for the modernization of traditional Chinese medicine. Yao Xue Xue Bao 2012; 47: 696-703. Chinese.

18 Zhao S, Li S. Network-based relating pharmacological and genomic spaces for drug target identification. PLoS One 2010; 5: e11764.

19 Li S, Zhang B, Zhang N. Network target for screening synergistic drug combinations with application to traditional Chinese medicine. BMC Syst Biol 2011; 5 Suppl 1: S10.

20 Li S, Zhang B, Jiang D, Wei Y, Zhang N. Herb network construction and co-module analysis for uncovering the combination rule of traditional Chinese herbal formulae. BMC Bioinformatics 2010; 11 Suppl 11: s6.

21 Wishart DS, Knox C, Guo AC, Cheng D, Shrivastava S, Tzur D, et al. DrugBank: a knowledgebase for drugs, drug actions and drug targets. Nucleic Acids Res 2008; 36: D901-6.

22 Hamosh A, Scott AF, Amberger JS, Bocchini CA, McKusick VA. Online mendelian inheritance in man (OMIM), a knowledgebase of human genes and genetic disorders. Nucleic Acids Res 2005; 33: D514-7.

23 Chen JY, Mamidipalli S, Huan T. HAPPI: an online database of comprehensive human annotated and predicted protein interactions. BMC Genomics 2009; 10 Suppl 1: S16.

24 Matthews L, Gopinath G, Gillespie M, Caudy M, Croft D, de Bono B, et al. Reactome knowledgebase of human biological pathways and processes. Nucleic Acids Res 2009; 37: D619-22.

25 Brown KR, Jurisica I. Online predicted human interaction database. Bioinformatics 2005; 21: 2076-82.

26 Aranda B, Achuthan P, Alam-Faruque Y, Armean I, Bridge A, Derow C, et al. The IntAct molecular interaction database in 2010. Nucleic Acids Res 2010; 38: D525-31. 
27 Keshava Prasad TS, Goel R, Kandasamy K, Keerthikumar S, Kumar $S$, Mathivanan S, et al. Human protein reference database - 2009 update. Nucleic Acids Res 2009; 37: D767-72.

28 Ceol A, Chatr Aryamontri A, Licata L, Peluso D, Briganti L, Perfetto L, et al. MINT, the molecular interaction database: 2009 update. Nucleic Acids Res 2010; 38: D532-9.

29 Lehne B, Schlitt T. Protein-protein interaction databases: keeping up with growing interactomes. Hum Genomics 2009; 3: 291-7.

30 Beuming T, Skrabanek L, Niv MY, Mukherjee P, Weinstein H. PDZBase: a protein-protein interaction database for PDZ-domains. Bioinformatics 2005; 21: 827-8.

31 GeneGo. Personal communication. MetaDrug Analysis Report. Prepared for NTP by GeneGo Inc. Tricolsan. Last updated on August 14, 2009.

32 Li S, Zhang ZQ, Wu LJ, Zhang XG, Li YD, Wang YY. Understanding ZHENG in traditional Chinese medicine in the context of neuroendocrine-immune network. IET Syst Biol 2007; 1: 51-60.

33 Wang Y, Liu Z, Li C, Li D, Ouyang Y, Yu J, et al. Drug target prediction based on the herbs components: the study on the multitargets pharmacological mechanism of qishenkeli acting on the coronary heart disease. Evid Based Complement Alternat Med 2012; 2012: 698531.

34 Wuchty S, Almaas E. Evolutionary cores of domain co-occurrence networks. BMC Evol Biol 2005; 5: 24.

35 Dennis G Jr, Sherman BT, Hosack DA, Yang J, Gao W, Lane HC, et al. DAVID: database for annotation, visualization, and integrated discovery. Genome Biol 2003; 4: P3.

36 Wixon J, Kell D. The Kyoto encyclopedia of genes and genomes KEGG. Yeast 2000; 17: 48-55.

37 Yu K, Gong Y, Lin Z, Cheng Y. Quantitative analysis and chromatographic fingerprinting for the quality evaluation of Scutellaria baicalensis Georgi using capillary electrophoresis. J Pharm Biomed Anal 2007; 43: 540-8.

38 Zhuang YS, Cai H, Liu X, Cai BC. Simultaneous determination of five main index components and specific chromatograms analysis in Xiaochaihu granules. Yao Xue Xue Bao 2012; 47: 84-7. Chinese.
39 Yang Y, Wang HJ, Yang J, Brantner AH, Lower-Nedza AD, Si N, et al. Chemical profiling and quantification of Chinese medicinal formula Huang-Lian-Jie-Du decoction, a systematic quality control strategy using ultra high performance liquid chromatography combined with hybrid quadrupole-orbitrap and triple quadrupole mass spectrometers. J Chromatogr A 2013; 1321: 88-99.

40 Brinks HL, Eckhart AD. Regulation of GPCR signaling in hypertension. Biochim Biophys Acta 2010; 1802: 1268-75.

41 Wang Y, Huang J, Li Y, Yang GY. Roles of chemokine CXCL12 and its receptors in ischemic stroke. Curr Drug Targets 2012; 13: 166-72.

42 Pini A, Mannaioni G, Pellegrini-Giampietro D, Passani MB, Mastroianni $\mathrm{R}$, Bani $\mathrm{D}$, et al. The role of cannabinoids in inflammatory modulation of allergic respiratory disorders, inflammatory pain and ischemic stroke. Curr Drug Targets 2012; 13: 984-93.

43 Bennett MV, Zukin RS. Electrical coupling and neuronal synchronization in the mammalian brain. Neuron 2004; 41: 495-511.

44 Zhang C, Li Y, Chen J, Gao Q, Zacharek A, Kapke A, et al. Bone marrow stromal cells upregulate expression of bone morphogenetic proteins 2 and 4, gap junction protein connexin-43 and synaptophysin after stroke in rats. Neuroscience 2006; 141: 687-95.

45 Nakase T, Fushiki S, Sohl G, Theis M, Willecke K, Naus CC. Neuroprotective role of astrocytic gap junctions in ischemic stroke. Cell Commun Adhes 2003; 10: 413-7.

46 Kozoriz MG, Bechberger JF, Bechberger GR, Suen MW, Moreno $\mathrm{AP}$, Maass $\mathrm{K}$, et al. The connexin43 C-terminal region mediates neuroprotection during stroke. J Neuropathol Exp Neurol 2010; 69: 196-206.

47 Zhang GB, Li QY, Chen QL, Su SB. Network pharmacology: a new approach for Chinese herbal medicine research. Evid Based Complement Alternat Med 2013; 2013: 621423.

48 Wang RZ, Lin DQ, Tong HF, Yao SJ. Molecular insights into the binding selectivity of a synthetic ligand DAAG to Fc fragment of IgG. J Mol Recognit 2014; 27: 250-9.

49 Rao SN, Head MS, Kulkarni A, LaLonde JM. Validation studies of the site-directed docking program LibDock. J Chem Inf Model 2007; 47: 2159-71. 